\title{
TITLE:
}

\section{Über den Gleichgewichtszustand Gemischter Salzlösung}

$\operatorname{AUTHOR}(S)$ :

Sasaki, Nobuji

\section{CITATION:}

Sasaki, Nobuji. Über den Gleichgewichtszustand Gemischter Salzlösung. Memoirs of the College of Science, Kyoto Imperial University. Series A 1925, 7(6): 361-368

ISSUE DATE:

1925-03-30

URL:

http://hdl.handle.net/2433/256684

RIGHT: 


\title{
Über den Gleichgewichtszustand Gemischter Salzlösung.
}

\author{
Von
}

Nobụi Sasaki.

(Eingegangen am 17. Januar 1924)

In der vorliegenden Abhandlung beabsichtige ich, den Gleichgewichtszustand einer aus zwei einwertigen gleichartigen Anionen und mehreren Kationen von verschiedener Wertigkeit bestehenden gemischten Lözung mathematisch unterzusuchen.

Als Beispiel denken wir uns eine Lösung, welche eine bestimmte Menge von Ferrichlorid, Bromnatrium, Chlornatrium und Salzsäure enthält. Im Gleichgewichte gibt es in der Lösung folgende Ionen und Ionengruppen :

1. Freie Ionen : $\mathrm{Na}^{*}, \mathrm{H}^{*}, \mathrm{Fe}^{\cdots}, \mathrm{Cl}^{\prime}, \mathrm{Br}^{\prime}$;
Anzahl
$b_{\circ} c_{\circ} \quad d_{\mathrm{o}} \quad a_{1} \quad a_{2}$
$a_{1}+a_{2}=a$

2. Ionengruppen von dem Typus $\mathrm{NaX}$ :

$$
\mathrm{NaCl}, \quad \mathrm{NaBr} \text {; }
$$
Anzahl
$b_{1}$
$b_{2}$
$b_{1}+b_{2}=b$

3. Ionengruppen von dem 'Typus $\mathrm{HX}$ :

$\mathrm{HCl}, \mathrm{HBr}$;
Anzahl
$c_{\mathrm{l}}$
$c_{2}$
$c_{1}+c_{2}=c$

4. Ionengruppen von dem Typus $\mathrm{FeX}^{*}$ :

$$
\mathrm{FeCl}^{*}, \quad \mathrm{FeBr}{ }^{\circ} \text {; }
$$
Anzahl
$d_{1}$
$d_{2}$
$d_{1}+d_{2}=d$ 
5. Ionengruppen von dem Typus $\mathrm{FeX}_{2}$ :

$\mathrm{FeCl}_{2}^{*}, \quad \mathrm{FeClBr}^{\circ}, \quad \mathrm{FeBr}_{2}^{\circ}$;
Anzahl
$e_{1}$
$e_{2}$
$e_{3}$
$e_{1}+e_{2}+e_{3}=e$

6. Ionengruppen von dem Typus $\mathrm{FeX}_{3}$ :

$\mathrm{FeCl}_{3}, \mathrm{FeCl}_{2} \mathrm{Br}, \mathrm{FeClBr}_{2}, \mathrm{FeBr}_{3}$;

$\begin{array}{llllll}\text { Anzahl } & f_{1} & f_{2} & f_{3} & f_{4} & f_{1}+f_{2}+f_{3}+f_{4}=f .\end{array}$

Wir wollen hier annehmen, dass sowohl Chlor- als auch Bromion elektrolytisch ein identisches Verhalten haben. Unter dieser Annahme sind die Grössen $b_{\circ}, c_{\mathrm{o}}, d_{\mathrm{o}}, a, b, c, d, e$ und $f$ durch die zugesetzten Mengen von Ferrichlorid, Bromnatrium, Chlornatrium und Salzsäure eindeutig bestimmt. Indem man daher die genannten Grössen als bekannt ansieht, wollen wir die Grössen $a_{1}, a_{2}, b_{1}, b_{2}, c_{1}, c_{2}, d_{1}, d_{2}, e_{1}, e_{2}, e_{3}$, $f_{1}, f_{2}, f_{3}$ und $f_{4}$ nach statistischer Methode berechnen.

Ist die Gesamtanzahl von Chlorion in der Lösung $m$, und die von Bromion $n$, so gelten die Beziehungen :

$$
\left.\begin{array}{c}
m=a_{1}+b_{1}+c_{1}+d_{1}+2 e_{1}+e_{2}+3 f_{1}+2 f_{2}+f_{3} \\
n=a_{2}+b_{2}+c_{2}+d_{2}+e_{2}+2 e_{3}+f_{2}+2 f_{3}+3 f_{4}
\end{array}\right\}
$$

Von den zwei Beziehungen (1) ist nur eine unabhängig, weil die andere aus den übrigen abgeleitet werden kann.

Wir wollen zunächst berechnen, in wieviel verschiedenen Weisen die angegebene Chlor-Bromionenverteilung aus $m$ Chlor-und $n$ Bromionen gewonnen werden kann. Aus $m$ Chlorionen kann man z. B. auf

$$
\frac{m P a_{1}}{(1 !)^{a_{1}}} \cdot \frac{1}{a_{1} !}
$$

verschiedene Weisen $a_{1}$ Chlorionen auswählen, und aus den gebliebenen 
Uber den Gleichgewichtszustand Gemischter Salzlösung.

$m-a_{1}$ Chlorionen auf

$$
\frac{\left(m-a_{1}\right) P b_{1}}{(1 !)^{b_{1}}} \cdot \frac{1}{b_{1} !}
$$

verschiedene Weisen $b_{1}$ Chlorionen auswählen, usw. Unten werden auf diese Weise berechnete Anzahl der Auswählungsweisen für einzelne Ionengruppen gegeben.

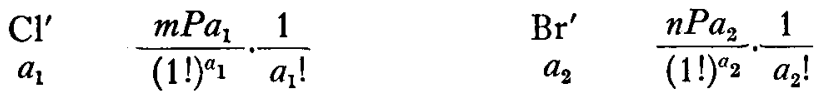

$$
\begin{aligned}
& \underset{b_{1}}{\mathrm{NaCl}} \quad \frac{\left(m-a_{1}\right) P b_{1}}{(1 !)^{b_{1}}} \cdot \frac{1}{b_{1} !} \quad \mathrm{NaBr} \quad \frac{\left(n-a_{2}\right) P b_{2}}{(1 !)^{b_{2}}} \cdot \frac{1}{b_{2} !} \\
& \underset{c_{1}}{\mathrm{HCl}} \quad \frac{\left(m-a_{1}-b_{1}\right) P c_{1}}{(1 !)^{c_{1}}} \cdot \frac{1}{c_{1} !} \quad \underset{c_{2}}{\mathrm{HBr}} \quad \frac{\left(n-a_{2}-b_{2}\right) P c_{2}}{(1 !)^{c_{2}}} \cdot \frac{1}{c_{2} !} \\
& \underset{d_{1}}{\mathrm{FeCl}^{*}} \frac{\left(m-a_{1}-b_{1}-c_{1}\right) P d_{1}}{(1 !)^{d_{1}}} \cdot \frac{1}{d_{1} !} \quad \underset{d_{2}}{\mathrm{FeBr}} \cdot \frac{\left(n-a_{2}-b_{2}-c_{2}\right) P d_{2}}{(1 !)^{d_{2}}} \cdot \frac{1}{d_{2} !} \\
& \underset{e_{1}}{\mathrm{FeCl}_{2}} \frac{\left(m-a_{1}-b_{1}-c_{1}-d_{1}\right) P 2 \mathrm{e}_{1}}{(2 !)^{e_{1}}} \cdot \frac{1}{e_{1} !} \\
& \underset{e_{2}}{\mathrm{FeClBr}} \quad \frac{\left(m-a_{1}-h_{1}-c_{1}-d_{1}-2 e_{1}\right) P e_{2}}{(1 !)^{e_{2}}} \cdot \frac{\left(n-a_{2}-b_{2}-c_{2}-d_{2}\right) P e_{2}}{(1 !)^{e_{2}}} \cdot \frac{1}{e_{2} !} \\
& \mathrm{FeBr}_{e_{3}}^{\mathrm{F}_{2}} \quad \frac{\left(n-a_{2}-b_{2}-c_{2}-d_{2}-e_{2}\right) P 2 e_{3}}{(2 !)^{r_{3}}} \cdot \frac{1}{e_{3} !} \\
& \underset{f_{1}}{\mathrm{FeCl}_{3}} \quad \frac{\left(m-a_{1}-b_{1}-c_{1}-d_{1}-2 e_{1}-e_{2}\right) P 3 f_{1}}{(3 !)^{f_{1}}} \cdot \frac{1}{f_{1} !} \\
& \underset{f_{2}}{\mathrm{FeCl}_{2} \mathrm{Br}} \frac{\left(m-a_{1}-b_{1}-c_{1}-d_{1}-2 e_{1}-e_{2}-3 f_{1}\right) P 2 f_{2}}{(2 !)^{f_{2}}} . \\
& \left.\frac{\left(n-a_{2}-b_{2}-c_{2}-d_{2}-e_{2}-2 e_{3}\right) P f_{2}}{(1 !)^{f_{2}}} \cdot \frac{1}{f_{2} !}\right)
\end{aligned}
$$


$\left.\begin{array}{rl}\mathrm{FeClBr}_{3} & \frac{\left(m-a_{1}-b_{1}-c_{1}-d_{1}-2 e_{1}-e_{2}-3 f_{1}-2 f_{2}\right) P f_{3}}{(1 !)^{f_{3}}} \cdot \\ \frac{\left(n-a_{2}-b_{2}-c_{2}-d_{2}-e_{2}-2 e_{3}-f_{2}\right) P 2 f_{3}}{(2 !)^{f_{3}}} \cdot \frac{1}{f_{3} !}\end{array}\right\}$

$\underset{f_{4}}{\mathrm{FeBr}_{3}} \quad \frac{\left(n-a_{2}-b_{2}-c_{2}-d_{2}-e_{2}-2 e_{3}-f_{2}-2 f_{3}\right) P 3 f_{4}}{(3 !)^{f_{4}}}, \frac{1}{f_{4} !}$

Das Produkt aus allen diesen Zahlen $\theta$ gibt die gesuchte Anzahl der verschiedenen Zusammens'ellungsweisen der oben gegebenen Ionenverteilung an :

$$
\theta=\frac{m ! n !}{a_{1} ! a_{2} ! b_{1} ! b_{2} ! c_{1} ! c_{2} ! d_{1} ! d_{2} ! e_{1} ! e_{2} ! e_{3} ! f_{1} ! f_{2} ! f_{3} ! f_{1} ! 2^{e_{1}} 2^{f_{3}} 3^{f_{1}} 3^{f_{4}} 2^{f}} .
$$

$\mathrm{Da} a_{1}, a_{2}$, usw. alle sehr gross sind, so kann man hier die Stirlingsche Formel

$$
n !=\sqrt{2 \pi n} n n^{n} e^{-n}
$$

anwenden. Durch Einsetzung der Formel erhält man

$$
\theta=\frac{A}{K},
$$

wobei

$$
\begin{aligned}
& A=\frac{m ! n ! e^{a+b+c+d+c+t}}{(\sqrt{2 \pi})^{15} 2^{f}},
\end{aligned}
$$

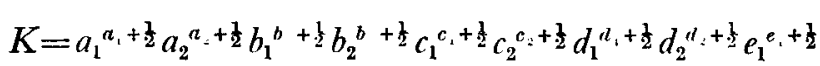

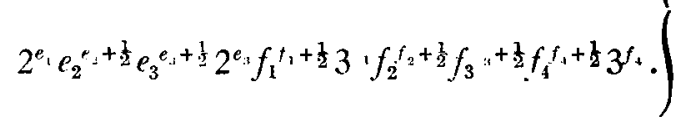

Wir wollen hier annehmen, dass im Gleichgewichte die wirkliche Ionenverteilung diejenige sein soll, welche die höchste Wahrscheinlichkeit hat, $d$. h. diejenige, welche den grössten Wert von $\theta$ hat. $\theta$ ist maximal, wenn $K$ minimal ist. Man gewinnt als die Maximum- bezw. Minimumbedingung 
Uber den Gleichgewichtszustand Gemischter Salzlösung.

$$
\begin{aligned}
\frac{\delta K}{K}= & \left(\log a_{1}+1+\frac{1}{2 a_{1}}\right) \delta a_{1}+\left(\log a_{2}+1+\frac{1}{2 a_{2}}\right) \delta a_{2}+\ldots . \\
& +\left(\log d_{2}+1+\frac{1}{2 d_{2}}\right) \delta d_{2}+\left(\log 2 e_{1}+1+\frac{1}{2 e_{1}}\right) \delta e_{1}+\left(\log e_{2}+1+\frac{1}{2 e_{2}}\right) \delta e_{2} \\
& +\left(\log 2 e_{3}+1+\frac{1}{2 e_{3}}\right) \delta e_{3}+\left(\log 3 f_{1}+1+\frac{1}{2 f_{1}}\right) \delta f_{1}+\left(\log f_{2}+1+\frac{1}{2 f_{2}}\right) \delta f_{2} \\
& +\left(\log f_{3}+1+\frac{1}{2 f_{3}}\right) \delta f_{3}+\left(\log 3 f_{4}+1+\frac{1}{2 f_{4}}\right) \delta f_{4}=0 .
\end{aligned}
$$

Andererseits erhält man aus Bedingung (2)

$$
\begin{aligned}
& \partial a_{2}=-\delta a_{1}, \delta b_{2}=-\delta b_{1}, \quad \delta c_{2}=-\delta c_{1}, \delta d_{2}=-\delta d_{1}, \\
& \delta e_{2}=-\delta e_{1}-\delta e_{3}, \partial f_{3}=-\delta f_{1}-\delta f_{2}-\delta f_{4} .
\end{aligned}
$$

Durch Einsetzung dieser Beziehungen und Vernachlässigung von der sehr kleinen Grösse $1 / 2 a_{1}$ gegen $\log a_{1}+1$ usw. erhält man

$$
\left.\begin{array}{c}
\frac{\delta K}{K}=\log \frac{a_{1}}{a_{2}} \delta a_{1}+\ldots+\log \frac{d_{1}}{d_{2}} \delta d_{1}+\log \frac{2 e_{1}}{e_{2}} \delta e_{1}+\log \frac{2 e_{3}}{e_{2}} \partial e_{3} \\
+\log \frac{3 f_{1}}{f_{3}} \delta f_{1}+\log \frac{f_{2}}{f_{3}} \delta f_{2}+\log \frac{3 f_{4}}{f_{3}} \delta f_{4}=0 .
\end{array}\right\}
$$

Aus Bedingung ( 1 ) folgt

$$
\delta a_{1}+\delta b_{1}+\delta c_{1}+\delta d_{1}+2 \delta e_{1}+\delta e_{2}+3 \delta f_{1}+2 \delta f_{2}+\delta f_{3}=0 ;
$$

aus Bedingung ( 2 ) folgt

$$
\begin{array}{rc}
\delta e_{1}+\delta e_{2}+\delta e_{3} & =0, \\
\delta f_{1}+\delta f_{2}+\delta f_{3}+\delta f_{4} & =0 .
\end{array}
$$

Durch Subtrahieren der letzten zwei Gleichungen von der ersten haben wir

$$
\delta a_{1}+\delta b_{1}+\delta c_{1}+\delta d_{1}+\delta e_{1}-\delta e_{3}+2 \delta f_{1}+\delta f_{2}-\delta f_{4}=0 .
$$

Multipliziert man ( 4 ) mit $\lambda$, einem unbestimmten Multiplikator und addiert dann zu (3), so erhält man

$$
\left.\frac{\partial K}{K}=\left(\log \frac{a_{i}}{a_{2}}+\lambda\right) \delta a_{1}+\ldots+\left(\log \frac{d_{1}}{d_{2}}+\lambda\right) \delta d_{1}+\left(\log \frac{2 e_{1}}{e_{2}}+\lambda\right) \delta e_{1}\right)
$$




$$
\begin{aligned}
& +\left(\log \frac{2 e_{3}}{e_{2}}-\lambda\right) \delta e_{3}+\left(\log \frac{3 f_{1}}{f_{3}}+2 \lambda\right) \delta f_{1}+\left(\log \frac{f_{2}}{f_{3}}+\lambda\right) \delta f_{2} \\
& +\left(\log \frac{3 f_{4}}{f_{3}}-\lambda\right) \delta f_{4}=0
\end{aligned}
$$

woraus folgt

$$
\begin{aligned}
& \log \frac{a_{1}}{a_{2}}+\lambda=0, \log \frac{b_{1}}{b_{2}}+\lambda=0, \log \frac{c_{1}}{c_{2}}+\lambda=0, \log \frac{d_{1}}{d_{2}}+\lambda=0, \\
& \log \frac{2 e_{1}}{e_{2}}+\lambda=0, \log \frac{2 e_{3}}{e_{2}}-\lambda=0, \\
& \log \frac{3 f_{1}}{f_{3}}+2 \lambda=0, \log \frac{f_{2}}{f_{3}}+\lambda=0, \log \frac{3 f_{4}}{f_{3}}-\lambda=0 .
\end{aligned}
$$

Die Gleichungen $\left(5_{1}\right)$ und $\left(5_{2}\right)$ und die letzten zwei von $\left(5_{3}\right)$ geben

$$
e^{-\lambda}=\frac{a_{1}}{a_{2}}=\frac{b_{1}}{b_{2}}=\frac{c_{1}}{c_{2}}=\frac{d_{1}}{d_{2}}=\frac{2 e_{1}}{e_{2}}=\frac{e_{2}}{2 e_{3}}=\frac{f_{2}}{f_{3}}=\frac{f_{3}}{3 f_{4}} .
$$

Die ersten zwei von $\left(5_{3}\right)$ geben

$$
\frac{3 f_{1}}{f_{3}}=\frac{f_{2}^{2}}{f_{3}^{2}}
$$

woraus folgt

$$
\frac{3 f_{1}}{f_{2}}=\frac{f_{2}}{f_{3}}
$$

Also haben wir

$$
e^{-\lambda}=\frac{a_{1}}{a_{2}}=\frac{b_{1}}{b_{2}}=\frac{c_{1}}{c_{2}}=\frac{d_{1}}{d_{2}}=\frac{2 e_{1}}{e_{2}}=\frac{e_{2}}{2 e_{3}}=\frac{3 f_{1}}{f_{2}}=\frac{f_{2}}{f_{3}}=\frac{f_{3}}{3 f_{1}},
$$

aus welcher Gleichung folgt

$$
e^{-\lambda}=\frac{a_{1}+b_{1}+c_{1}+d_{1}+2 e_{1}+e_{2}+3 f_{1}+2 f_{2}+f_{3}}{a_{2}+b_{2}+c_{2}+d_{2}+e_{2}+2 e_{3}+f_{2}+2 f_{3}+3 f_{4}}
$$

Daher ist $e^{-\lambda}$ gemäss (1) gleich $\frac{m}{n}$.

Schliesslich haben wir 


$$
\frac{m}{n}=\frac{a_{1}}{a_{2}}=\frac{b_{1}}{b_{2}}=\frac{c_{1}}{c_{2}}=\frac{d_{1}}{d_{2}}=\frac{2 e_{1}}{e_{2}}=\frac{e_{2}}{2 e_{3}}=\frac{3 f_{1}}{f_{2}}=\frac{f_{2}}{f_{3}}=\frac{f_{3}}{3 f_{4}} .
$$

Aus Gleichungen (2) und (7) ergibt sich die Anzahl der Ionengruppen :

$$
\begin{array}{rlrl}
a_{1} & =\frac{m}{m+n} a, & a_{2}=\frac{n}{m+n} a, \\
b_{1} & =\frac{m}{m+n} b, & b_{2} & =\frac{n}{m+n} b, \\
c_{1} & =\frac{m}{m+n} c, & c_{2} & =\frac{n}{m+n} c, \\
d_{1} & =\frac{m}{m+n} d, & d_{2} & =\frac{n}{m+n} d, \\
e_{1}=\frac{m^{2}}{(m+n)^{2}} e, & e_{2}=\frac{2 m n}{(m+n)^{2}} e, & e_{3}=\frac{n^{2}}{(m+n)^{2}} e_{i} \\
f_{1}=\frac{m^{3}}{(m+n)^{3}} f, \quad f_{2}=\frac{3 m^{2} n}{(m+n)^{3}} f, \quad f_{3}=\frac{3 m n^{2}}{(m+n)^{3}} f, \quad f_{4}=\frac{n^{3}}{(m+n)^{3}} f .
\end{array}
$$

Dass diese Verteilung von Chlor- und Bromionen die höchste Wahrscheinlichkeit besitzt, kann folgenderweise bewiesen werden.

Wie oben erhalten, ist

$$
\delta K=K\left[\left(\log a_{1}+1+\frac{1}{2 a_{1}}\right) \delta a_{1}+\ldots \ldots+\left(\log 3 f_{4}+1+\frac{1}{2 f_{4}}\right) \delta f_{4}\right] .
$$

Folglich ist

$$
\delta^{2} K=\frac{(\delta K)^{2}}{K}+K\left[\left(\frac{1}{a_{1}}-\frac{1}{2 a_{1}^{2}}\right)\left(\delta a_{1}\right)^{2}+\ldots \ldots+\left(\frac{1}{f_{4}}-\frac{1}{2 f_{4}^{2}}\right)\left(\delta f_{4}\right)^{2}\right]
$$

woraus man gleich ersiht, dass $\delta^{2} K$ stets positiv ist. Also ist $K \mathrm{mi}-$ nimal und $\theta$ deshalb maximal für die erhaltenen Werte der Ionengruppen. Diese Ionenverteilung ist sonach die wahrscheinlichste. 
368 N. Sasaki: ["lber den Gleichgewichtszustand Gemischter Salzlösung.

\section{Zusammenfassung.}

Es wurden am Beispiel der gemischten Lösung von $\mathrm{FeCl}_{3}, \mathrm{NaCl}$, $\mathrm{HCl}$ und $\mathrm{NaBr}$ die Konzentrationen aller möglichen Ionen and Ionengruppen nach statistischer Methode berechenet.

Es ist mir eine angenehme Pflicht, meinem hochverehrten Lehrer Herrn Prof. Dr. M. Chikashige für seine ständige Unterstützung und auch Herrn Prof. Dr. M. Sono für seine freundliche Durchsicht an dieser Stelle meinen herzlichsten Dank auszusprechen. 